\title{
Anisotropic rupture of polymer strips driven by Rayleigh instability
}

\author{
Jeong-yong Park \\ School of Chemical and Biological Engineering, Seoul National University, Seoul 151-742, Korea \\ Kahp Y. Suh \\ School of Mechanical and Aerospace Engineering, Seoul National University, Seoul 151-742, Korea \\ and Institute of Advanced Machinery and Design, Seoul National University, Seoul 151-742, Korea \\ Soon-min Seo and Hong H. Lee ${ }^{\text {a) }}$ \\ School of Chemical and Biological Engineering, Seoul National University, Seoul 151-742, Korea
}

(Received 20 February 2006; accepted 27 April 2006; published online 7 June 2006)

\begin{abstract}
We demonstrate that the separated polymer strips of micro- and sub-micro-length-scales rupture anisotropically along the strip direction, resulting in the formation of distinctly observable, regularly spaced polymer drops. The wavelength of the polymer drops and the surface tension dependence of the rupture behavior are found to be well represented by a relationship derived on the basis of Rayleigh instability. The period is proportional to the square root of the cross-sectional area of the strip and the proportionality constant depends on the contact angle. The rupture of polymer strips into polymer blocks instead of drops, which result when annealed with physically confining walls in place, is found to be well described by the same relationship. (C) 2006 American Institute of Physics. [DOI: 10.1063/1.2206580]
\end{abstract}

\section{INTRODUCTION}

Drop formation and thermal stability of thin liquid films on partially wetted substrate have attracted much attention because of fundamental interest in basic wetting/dewetting phenomena, ${ }^{1}$ stability of nanojets, ${ }^{2}$ nanowire fragmentation, ${ }^{3}$ micro/nanoscale patterning, ${ }^{4-6}$ and various industrial applications such as fiber coating ${ }^{7}$ and microelectronic device manufacturing. ${ }^{8}$ Since Rayleigh described the capillary instability of inviscid fluids, ${ }^{9}$ extensive theoretical and experimental works have been carried out towards verifying and understanding the surface tension driven breakup of thin liquid films in various disciplines (e.g., hydrodynamics and materials science).

Until now, however, most studies have dealt with the dewetting behavior of two-dimensional polymer films. These studies revealed that there are two classical dewetting routes in the thin films: a process whereby film rupture is induced by nucleated holes ${ }^{10}$ and a different pathway through which van der Waals intermolecular forces amplify thermal fluctuations (often termed "spinodal dewetting"). ${ }^{11}$ It has been reported that the spinodal dewetting is effective for very thin films of polymer (typically $<10 \mathrm{~nm}$ ) of low molecular weight $\left(<10^{4}\right){ }^{11}$ On the other hand, nucleation of holes takes place for intermediate film thickness $(100 \mathrm{~nm}-1 \mu \mathrm{m})$ that is accelerated by the presence of small particles (i.e., dust) or imperfections on the surface. ${ }^{12}$

While the literature is abundant on dewetting of flat film, there have been few studies on the rupture behavior of one dimensional polymer strips. To gain an understanding of the rupture behavior, we specifically devised an experiment where separated polymer strips rupture upon heating. Our

\footnotetext{
${ }^{a)}$ Author to whom correspondence should be addressed. Electronic mail: honghlee@snu.ac.kr
}

major motivation was to find whether the geometry of polymer film affects the rupturing behavior of the polymer. We found that the polymer strips rupture anisotropically. The period of strip fragments was smaller than that of the drops formed by isotropic dewetting of flat polymer film and well correlated with the length scale corresponding to the distance between two contact lines of polymer strip, which is similar to the well-known rupture behavior of a narrow, moving liquid thread or rim. The results would be the first experimental demonstration that the Rayleigh instability of immobile polymer thread can be directly applied to micro- or sub-microlength-scale.

\section{EXPERIMENTAL METHOD}

For the experimental setup, we used a poly(dimethylsiloxane) (PDMS) (Sylgard 184, Dow Corning) mold that has a planar surface with recessed patterns by casting PDMS against the master mold that was prepared by photolithographic method on a silicon wafer $(100) .{ }^{13}$ The mold has line-and-space patterns varying in width from $800 \mathrm{~nm}$ to $2.5 \mu \mathrm{m}$ with a step height of $600 \mathrm{~nm}$. The mold with the pattern is placed on the surface of a polymer layer spin coated onto a substrate and then heated above the glass transition temperature of the polymer. Capillary force then allows the polymer melt to fill up the void space of the channels formed between the mold and the polymer, thereby generating a laterally confined geometry. ${ }^{14}$ With the polymer strips thus formed, annealing was carried out in the absence of PDMS walls, as illustrated in the schematic diagram in Fig. 1. The patterns resulting from the annealing were examined by an optical microscope (BX60, Olympus Co., Ltd., Japan), atomic force microscopy (AFM) (Dimension 

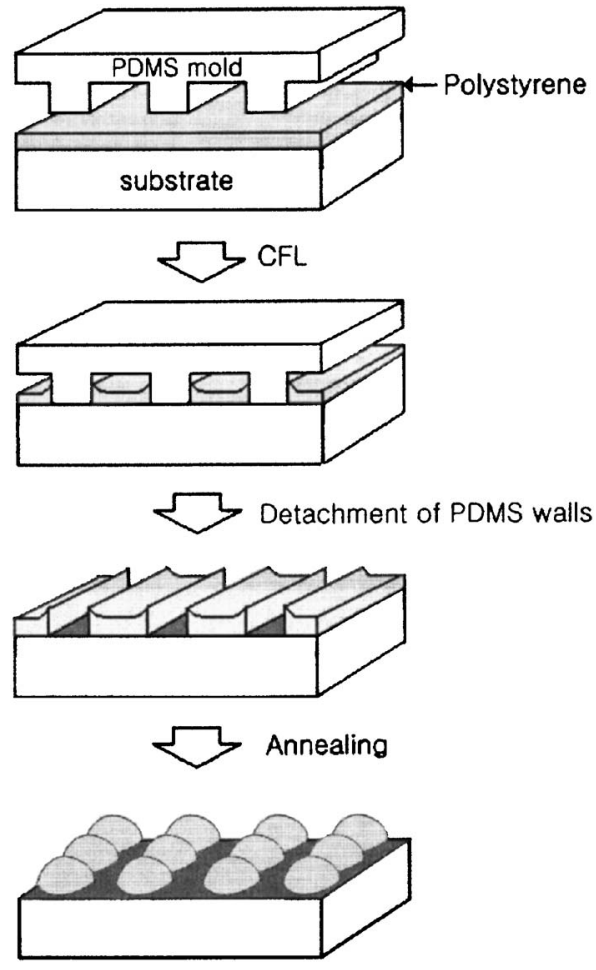

FIG. 1. Schematic diagram of the experimental procedure.

3100, Veeco Instruments, Inc., USA), and scanning electron microscopy (SEM) (XL30FEG, Philips Electron Co., Netherlands).

For the polymer, we used commercial polystyrene (PS) (molecular weight $=24.7000, T_{g}=101{ }^{\circ} \mathrm{C}$, Polymer Source, Inc., Canada). Silicon wafer and $\mathrm{SiN}_{x}$ deposited silicon wafer that were used as the substrate were cleaned by ultrasonic treatment in trichloroethylene and methanol for $5 \mathrm{~min}$ each and dried in nitrogen. The native oxide of the silicon wafer was not removed and thus would exist on the surface. Polymer films were spin coated onto the substrate to thicknesses of 70, 92, and $110 \mathrm{~nm}$ on Si wafer, and 87, 96, and $108 \mathrm{~nm}$ on $\mathrm{SiN}_{x}$ deposited wafer, respectively, as confirmed by ellipsometry (Nanospec AFT/4150, Nanometrics, Inc., USA; L2W15S830, Gaertner Scientific Co., USA).

\section{RESULT AND DISCUSSION}

It is noted that a physical separation of polymer strips is a critical factor for the formation of anisotropic polymer fragments. A typical example in Fig. 2 reveals the importance of the physical separation. There are two regions in Fig. 2(a): the left region where physically separated polymer strips were originally present and the right where a continuous film was present. The magnified version of Fig. 2(a) in 2(b) clearly shows that the isolated polymer strips of $2 \mu \mathrm{m}$ wide on the left ruptured anisotropically, leading to the formation of regularly spaced polymer drops. By contrast, the two-dimensional polymer film on the right dewetted isotropically, leading to a pattern corresponding to the well-known rim instability. ${ }^{10,15,16}$ No residual layer was found on the space regions between the adjacent strips, which is a major advantage of our patterning method called capillary force lithography (CFL). ${ }^{14}$ It would be difficult or costly to fabricate such polymer strips with other patterning methods.

This finding provides a fundamental insight into how the rupture mechanism depends on the geometry of the film. As can be seen from Figs. 2(a) and 2(b), the wavelength of the fragments resulting from the polymer strip, which has onedimensional geometry and is separated from the other, is much smaller than that of the nonseparated flat polymer film. The height of the isolated strip is approximated to be two times the thickness of the initial film since an equal line-andspace pattern was used and complete capillary rise took place, leading to a height of $\sim 184 \mathrm{~nm}$ for the initial thickness of $92 \mathrm{~nm}$ in Figs. 2(a) and 2(b). According to the conventional capillary wave model, the wavelength $\lambda$ is proportional to the square of the initial film thickness $h_{0}$, i.e., $\lambda$ $\propto h_{0}^{2}$, which does not agree with our experimental results. On the other hand, the wavelength of the flat film was in good agreement with the value predicted by the capillary wave theory, suggesting that two different rupture mechanisms coexist in this experiment. We hypothesize that the intermolecular forces are the major fact in the rupture of the flat film (capillary wave model of a thin flat film) but the interfacial tension along the strip direction is responsible for the rupture of the isolated polymer strips (Rayleigh rupture model of a liquid thread) since the effects of intermolecular forces would be negligible in this thickness range.

Typical optical and cross-sectional SEM images of the separated polymer drops formed by the rupture of polymer strips are shown in Figs. 2(c) and 2(d), respectively. The width of PS strip was $2 \mu \mathrm{m}$ and the initial film thickness was $70 \mathrm{~nm}$. After the drop formation, the height of the drop increased to $\sim 450 \mathrm{~nm}$ due to mass transport and there was little polymer left in the region between the drops. As the height of the PDMS mold was $600 \mathrm{~nm}$, the effects of the ceiling of the mold could be ruled out for polymer drops.

To examine and compare the transient rupture morphology of PS strips, we carried out optical microscope measurements of the typical transient morphologies of PS strips for a $2 \mu \mathrm{m}$ line-and-space pattern in the absence of the PDMS walls [Figs. 3(a)-3(d)]. The onset of the instability needed a certain incubation time depending on the film thickness, channel width, and annealing temperature. To reduce the time scale for observation, the samples were annealed at $160{ }^{\circ} \mathrm{C}$. The initial film thickness was about $92 \mathrm{~nm}$ and the height of the PS strips formed was about $185 \mathrm{~nm}$. After CFL, a meniscus was observed on the upper side of the protruding part of the polymer, a signature of the capillary rise, because the polymer did not touch the ceiling of the mold. Also no residual layer was found in the spaces between the adjacent strips and thus the substrate surface was clearly exposed. Up to $30 \mathrm{~min}$, there was no observable change in the polymer strip pattern as shown in Fig. 3(a). As time went on, the meniscus disappeared and the rectangular shape of polymer strip was transformed into a rim or semicircular shape [Fig. $3(\mathrm{e})$. And then sinusoidal undulations occurred in the strip direction, indicating that the polymer surface fluctuates sinusoidally [Fig. 3(b)]. The strips started to rupture when an- 

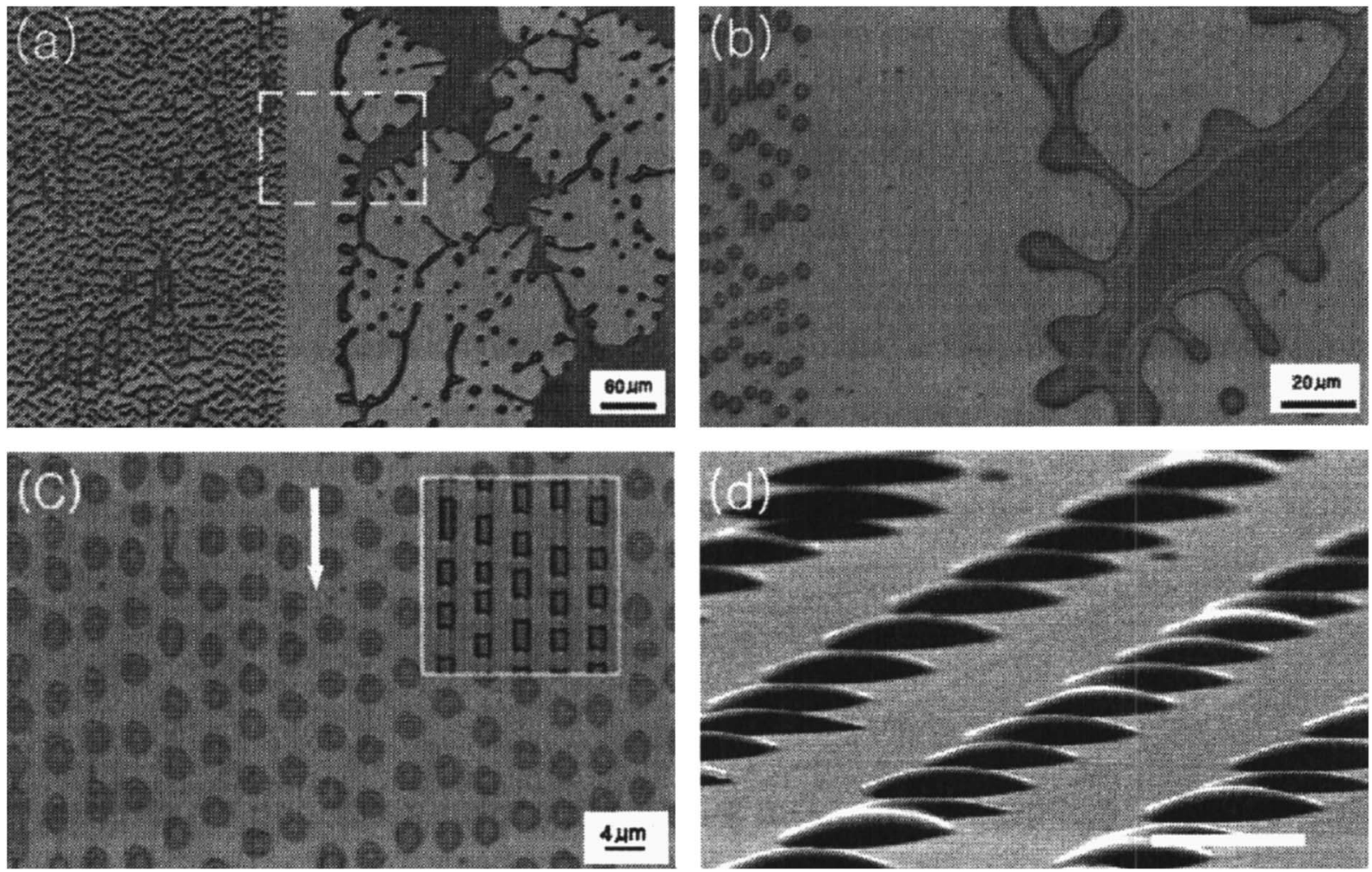

FIG. 2. (a) An optical image of drop formation of PS strips in the absence of the PDMS walls: on the left, $2 \mu \mathrm{m}$ strips were originally present and on the right an unconfined flat film. The initial film thickness was about $92 \mathrm{~nm}$. (b) Magnified image of the boxed region in (a). (c) An optical image of separated drops of $2 \mu \mathrm{m}$ wide polymer strips that resulted in the absence of PDMS walls. The inset shows the separated block formation in the same strips that resulted when confined by PDMS walls (from Ref. 20). The arrow indicates the direction of long axis of the polymer strips (d) A SEM cross-sectional view of (c). The scale bar represents $2 \mu \mathrm{m}$. The initial thickness is $70 \mathrm{~nm}$ and the samples were annealed at $160^{\circ} \mathrm{C}$.

nealed further [Fig. 3(c)]. Finally, the sinusoidal deformation resulted in the rupture of the PS strips with a distinctly observable period [Fig. 3(d)].

Interestingly, fluctuations shown in Figs. 3(a)-3(d) are very similar to those known in the rupture of a long, cylinder such as a liquid thread or a rim. ${ }^{3,9,15-19}$ The long cylindrical geometry has a characteristic that the length scale in one direction is much longer than that in the other, which is very similar to the isolated polymer strip used in our experiment. The theory of Rayleigh instability predicts that a long, continuous cylinder ruptures into separate drops to minimize the interfacial energy [Fig. 3(f)].

To explain our observations quantitatively, we derive a simple relationship based on rim instability because the polymer strips transform into a rim shape as shown in Fig. 3(e). A linear stability analysis yields the fastest growing mode $\lambda$ for a surface fluctuation during the initial stage of the fragmentation. The wavelength $\lambda$ is known to be directly proportional to the half-width of the rim, $x_{0}$ or $\lambda \sim 8 x_{0}{ }^{15}$ When an initially rectangular strip with width $L$ and thickness of $h$ is transformed into a rim, the cross-sectional area $\mathrm{A}$ is the same and we have

$$
A=L h \equiv R_{0}^{2}[\theta-\sin \theta \cos \theta]
$$

where $R_{0}$ is the constant radius of curvature of PS rim and $\theta$ is the contact angle, as shown in Fig. 4. Then the half-width of the rim $x_{0}$ can be written as follows:

$$
x_{0}=R_{0} \sin \theta=\frac{\sin \theta}{\sqrt{\theta-\sin \theta \cos \theta}} \sqrt{L h},
$$

where Eq. (1) was used for $R_{0}$, Therefore, the wavelength $\lambda$ is given by

$$
\lambda \sim \frac{8 \sin \theta}{\sqrt{\theta-\sin \theta \cos \theta}} \sqrt{L h} .
$$

This relationship states that the number of drops formed in a given strip is inversely proportional to the square root of the cross-sectional area of the strip. It also shows that the proportionality constant depends on the surface tension or the contact angle. This dependence is an essential feature of Rayleigh instability that is driven by surface tension. To verify the results, we carried out experiments with the PS films patterned on both $\mathrm{Si}$ wafer substrate and $\mathrm{SiN}_{x}$ deposited silicon wafer substrate. Shown in Fig. 4(a) is a plot of the number density of drops in $200 \mu \mathrm{m}$ long strip on the $\mathrm{Si}$ substrate (solid line through filled rectangles) and on the $\mathrm{SiN}_{x}$ substrate (dotted line through crosses) as a function of the cross-sectioned area of the strip, assuming that the height is two times the initial film thickness. Various channel widths $(0.8,0.9,1,1.5$, and $2 \mu \mathrm{m}$ or $2.5 \mu \mathrm{m})$ with different film thicknesses were used in the experiment. Fitting the experimental data in Fig. 4(a) yielded a slope of about $-0.5(-0.55$ on $\mathrm{Si}$ and -0.48 on $\mathrm{SiN}_{x}$ ), which coincides with the slope predicted by Eq. (3), and the intercepts gave proportionality constants of 11.44 on $\mathrm{Si}$ surface and 10.33 on $\mathrm{SiN}_{x}$ surface. 


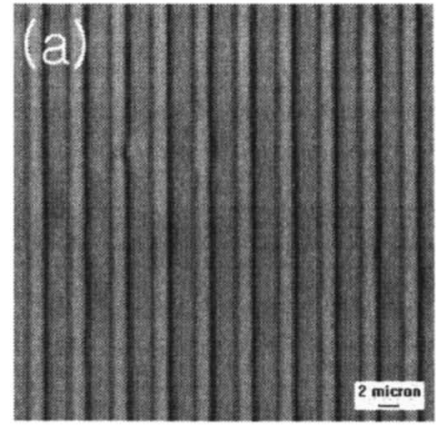

(e)

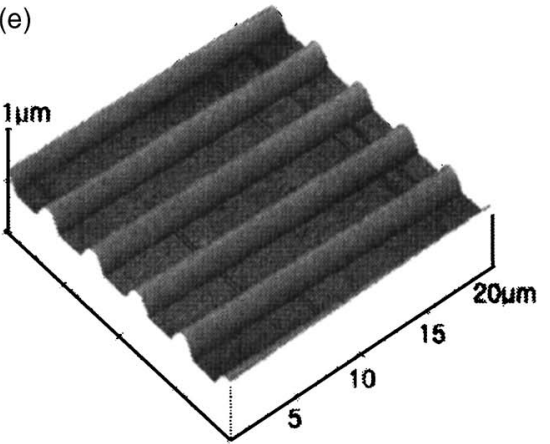

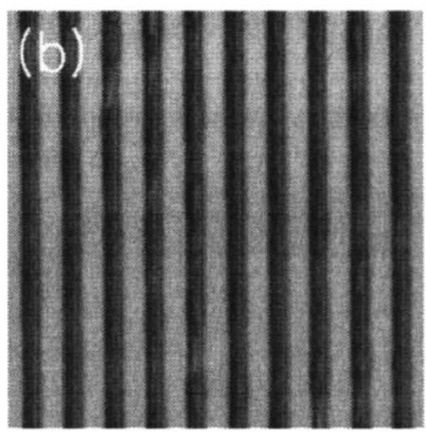
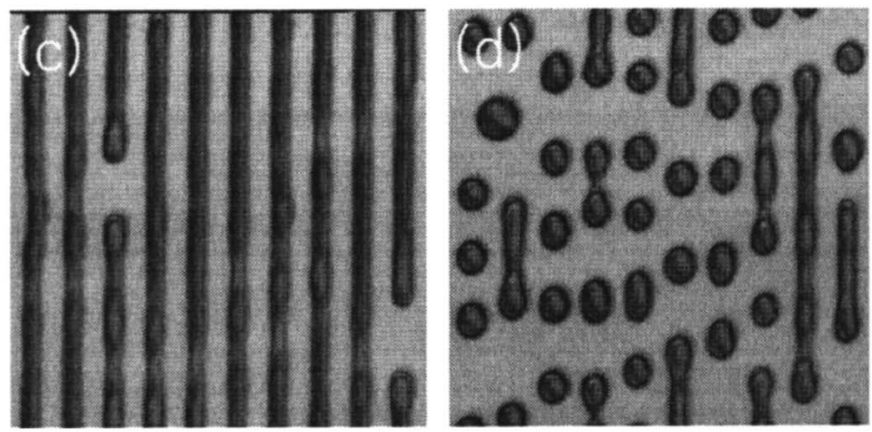

(f)

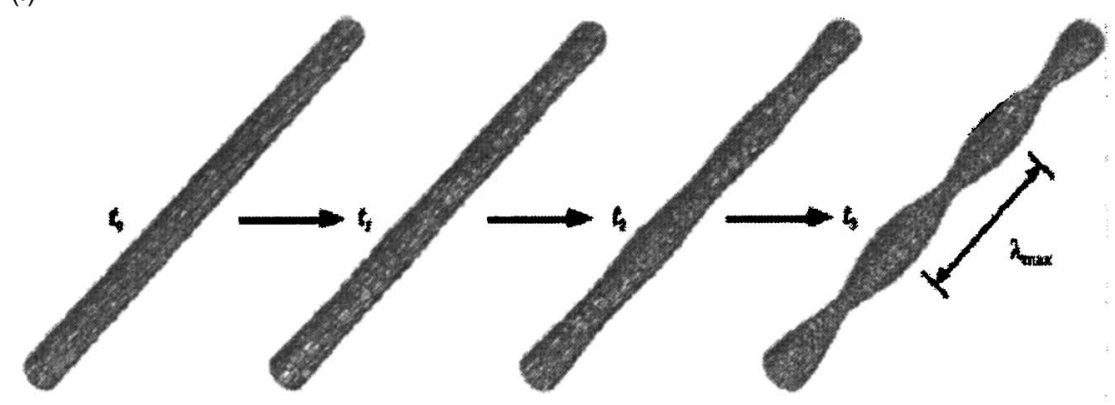

FIG. 3. Optical microscopy images of the transient morphologies of $2 \mu \mathrm{m}$ wide polystyrene strips in the absence of PDMS walls. Pictures were taken after (a) 0.5 , (b) 1, (c) 2, and (d) $4 \mathrm{~h}$, respectively. The initial film thickness was about $92 \mathrm{~nm}$. (e) three-dimensional AFM image of PS strip. The annealing time is about 50 min just before forming the surface fluctuation. (f) Morphological evolution associated with Rayleigh instability (from Ref. 17).

These values compare with 12.42 on Si surface $\left(\theta=34^{\circ}\right)$ and 10.59 on $\operatorname{SiN}_{x}$ surface $\left(\theta=45^{\circ}\right)$ that can be calculated from Eq. (3). These results reveal that a simple Rayleigh instability can accurately describe the anisotropic rupture of polymer strips of micro- and sub-micro-length-scales.

Recently, we reported anisotropic hole formation in thin polymer films laterally confined by PDMS walls. ${ }^{20}$ It was found that regularly spaced holes were initially nucleated on the polymer surface and then grew with the aid of the confining walls, resulting in the formation of distinctly observable, regularly spaced blocks of the strip [see the inset of Fig. 2(c)]. It was also found that the dependence of hole density on the film thickness deviated severely from the conventional capillary wave model.

We carried out the same experiment but this time annealing with PDMS walls still in place after CFL. Then we applied the theory of Rayleigh instability to the block formation by rupture of PS strips. To our surprise, the power index was also determined to be around -0.5 as shown in Fig. 5(b), which is fairly close to the value predicted by Eq. (3). The proportionality constants obtained from the experimental results in the figure were 11.73 (on $\mathrm{Si}$ surface) and 10.83 (on $\mathrm{SiN}_{x}$ surface), which are also fairly close to the calcu-

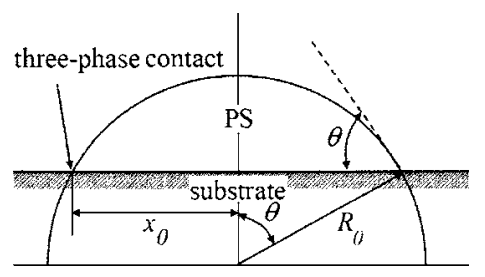

FIG. 4. Cross-sectional shape of a long cylindrical PS strip and its geometry. lated values given above. These results show that while the kinetic pathways are different, the same Rayleigh instability applies to the rupture of polymer strips even when the strips are confined by PDMS walls.

The reason for the hole formation instead of the sinusoidal undulations in the presence of PDMS walls could be attributed to a physical binding of the strip to the PDMS wall. It appears that the fluctuation is substantially suppressed for the laterally confined PS strips such that it would be much easier for the strip to be ruptured by means of hole formation and growth. Once the holes are initiated, they can grow with the aid of PDMS walls, resulting in the formation of regularly spaced polymer fragments. This hole formation and growth is also similar to that of the rim instability of a thin polymer film. Thus, the rupture appears to proceed in such a way that the energy cost could be minimized. This issue is currently under investigation.

A recent theoretical study provides a clue as to how the confinement affects the final morphology of a polymer

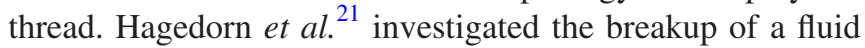
thread in a confined geometry and found that there is a transition in the late-stage morphology between spherical droplets and plugs depending on the degree of confinement. For weak confinement, spherical capsules were mostly found whereas polymer plugs or blocks were found for strong confinement, which agrees well with our experiment. However, as the geometry of their system was a fluid thread surrounded by a cylindrical tube, hole formation was not predicted by the simulations, which appears to be restricted to our experiment.

In summary, we have demonstrated that isolated polymer strips rupture in an anisotropic manner with a certain period. 

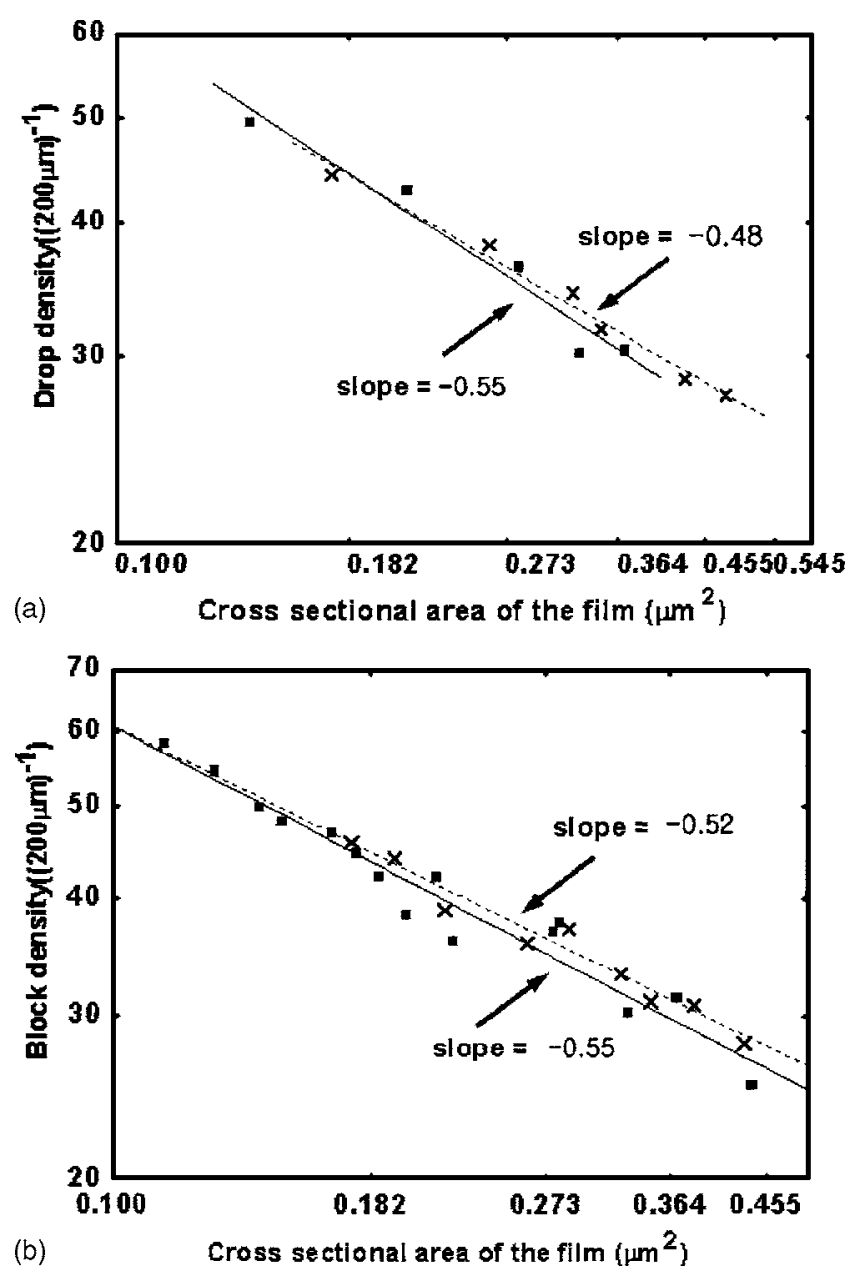

FIG. 5. Double logarithmic plot of the number density of PS fragments in $200 \mu \mathrm{m}$ long strip as a function of the cross-sectional area $(L h)$ of the strip. (a) Polymer drops formed upon annealing at $160{ }^{\circ} \mathrm{C}$. (b) polymer blocks formed upon annealing at $160{ }^{\circ} \mathrm{C}$ in the presence of PDMS walls [( $\left.\mathbf{\square}\right)$ solid lines are for $\mathrm{Si}$ surface and $(\times)$ dotted lines are for $\mathrm{SiN}_{x}$ surface]. An average was taken over more than ten strips for the number density. The height of the strip was assumed to be two times the initial film thickness.

The rectangular strip transforms initially into a rim shape upon annealing and then ruptures into regularly spaced polymer drops. An analysis based on Rayleigh instability shows that the period is proportional to the square root of the crosssectional area and the proportionality constant depends on the surface tension or the contact angle, an essential feature of Rayleigh instability driven by the surface tension. Experi- mental results obtained on two different surfaces have been shown to be well represented by the theoretical description, including the surface tension dependence of the anisotropic rupture. While the kinetic pathways are different for the anisotropic rupture of polymer strips in the presence of PDMS walls, which result in the formation of regularly spaced polymer blocks instead of polymer drops, the same theory has been found to be applicable. The experiment reported here demonstrates that Rayleigh instability could even be applied to the breakup of an immobile polymer thread of micro- and sub-micro-length-scales regardless of the confinement. The experimental approach and the theoretic result would be useful for a wide range of polymer films in a confined geometry.

\section{ACKNOWLEDGMENT}

K.Y.S. is grateful for the financial support from the Micro Thermal Research Center of Seoul National University.

${ }^{1}$ M. Schick, in Liquids at Interfaces, Proceedings of the Les Houches Summer School, Session No. 48, edited by J. Charvolin, J. F. Joanny, and J. Zinn-Justin (Elsevier, Amsterdam, 1990).

${ }^{2}$ M. Moseler and U. Landman, Science 289, 1165 (2000).

${ }^{3}$ M. E. Toimil Molares, A. G. Balogh, T. W. Cornelius, R. Neumann, and C. Trautmann, Appl. Phys. Lett. 85, 5337 (2004).

${ }^{4}$ S. Herminghaus, K. Jacobs, K. Mecke, J. Bischof, A. Fery, M. Ibn-Elhaj, and S. Schlagowski, Science 282, 916 (1998).

${ }^{5}$ A. M. Higgins and R. A. L. Jones, Nature (London) 404, 476 (2000).

${ }^{6}$ M. Gleiche, L. F. Chi, and H. Fuchs, Nature (London) 403, 173 (2000).

${ }^{7}$ D. Quere, J. D. Meglio, and F. Brochard-Wyart, Science 249, 1256 (1990).

${ }^{8}$ D. J. Hayes, W. R. Cox, and M. E. Grove, J. Electron. Manuf. 8, 209 (1998).

${ }^{9}$ L. Rayleigh, Proc. London Math. Soc. 10, 4 (1879).

${ }^{10}$ G. Reiter, Phys. Rev. Lett. 68, 75 (1992).

${ }^{11}$ R. Xie, A. Karim, J. F. Douglas, C. C. Han, and R. A. Weiss, Phys. Rev. Lett. 81, 1251 (1998).

${ }^{12}$ L. Leger and J. F. Joanny, Rep. Prog. Phys. 55, 431 (1992).

${ }^{13}$ Y. Xia, J. A. Rogers, K. E. Paul, and G. M. Whitesides, Chem. Rev. (Washington, D.C.) 99, 1823 (1999).

${ }^{14}$ K. Y. Suh, Y. S. Kim, and H. H. Lee, Adv. Mater. (Weinheim, Ger.) 13, 1386 (2001).

${ }^{15}$ F. Brochard-Wyart and C. Redon, Langmuir 8, 2324 (1992); O. Karthaus, L. Grasjo, N. Maruyama, and M. Shimomura, Chaos 9, 308 (1999).

${ }^{16}$ G. Reiter and A. Sharma, Phys. Rev. Lett. 87, 166103 (2001).

${ }^{17}$ A. M. Glaeser, Interface Sci. 9, 65 (2001).

${ }^{18}$ M. S. McCallum, P. W. Voorhees, M. J. Miksis, S. H. Davis, and H. Wong, J. Appl. Phys. 79, 7604 (1996).

${ }^{19}$ J. Eggers, Rev. Mod. Phys. 69, 865 (1997).

${ }^{20}$ K. Y. Suh and H. H. Lee, J. Chem. Phys. 115, 8204 (2001).

${ }^{21}$ J. G. Hagedorn, N. S. Martys, and J. F. Douglas, Phys. Rev. E 69, 056312 (2004). 